\title{
Investigations on generation of very low side lobe difference patterns for EMC applications
}

\author{
T A N S N Varma ${ }^{1,}$ Dr. G.S.N.Raju ${ }^{2}$ \\ ${ }^{1}$ Research scholar. ${ }^{2}$ Professor, \\ Department of ECE, Andhra University, Visakhapatnam-530003.
}

\begin{abstract}
Pattern synthesis is one of the most important aspects in antenna design. Arrays are more flexible to produce desired radiation characteristics. Difference patterns are usually generated with conventional techniques and there is less control on sidelobes. In view of this, techniques like genetic algorithm, particle swarm optimization and simulated annealing are applied to synthesize and produce such patterns optimally. The simulated patterns are produced for different array configurations and the patterns are presented in u domain.
\end{abstract}

Keywords: Difference pattern, Sidelobe reduction, Antenna Array, Genetic Algorithm, Particle Swarm Optimization and Simulated Annealing

\section{Introduction}

The radiation pattern structure is one of the most important specifications for the design of the antennas in all communication and radar applications [1]. Radars are highly effective in ascertaing the location of remote objects, but accuracy is greatly reduced when a radar is jammed. The difference pattern consists of a null in the boresight direction with two major lobes adjacent to null. The null in the difference pattern is suppressing the jamming source and tracking accuracy was improved [2].

The sum and difference patterns are essential in IIF radars, remote sensing radars and airport surveillance radars. The sum patterns are generated by several methods but the methods of generation of difference patterns are limited. In difference patterns, the depth of the null is significant and the difference slope is not enough for many applications. It is required to produce them for optimum performance. Generally the difference pattern is created by simultaneously exciting directional and Omni-directional antennas. In this method it is difficult to obtain deep null and high difference slope but for fine angular tracking difference pattern should have deep null at boresight and high difference slope for accurate angular tracking.

Arrays provide a greater number of parameters to generate the difference pattern such as geometrical configuration of an overall array, relative displacement between elements, excitation of individual radiating element and relative pattern of individual elements [3]. The excitation may consist of amplitude distribution as well as phase distribution. For scan applications, the phase distribution is not considered but for scanning applications both phase and amplitude distribution is considered.

Bayliss [4] synthesized the difference pattern for continuous line source but was not able to find a limiting form for the ideal difference pattern. R.S.Elliott [5] proposed a design technique which yields difference pattern with arbitrary sidelobe topographies together with the requisite continuous aperture distributions. Yanchang, G. et.al. [6] proposed a new design method which determines the aperture distribution for difference pattern from Taylor distribution. The peak sidelobe level in the difference pattern can be easily controlled using this method.

A method of using dolph-chebyshev distribution for difference pattern is developed in the paper by Yanchan Guo et. al. [7].Salvatore caorsi et. al. [8] discussed the difference patterns by hybrid differential evolution method in which subarray configurations is adopted and excitations of the difference patterns are approximately determined. The patterns reported has sidelobe level (SLL) around $-30 \mathrm{~dB}$.

The present work is focused on generation of difference patterns with deep null in boresight and very low sidelobe levels. In the last few years, a large interest has been devoted to the exploitation of global optimization techniques in electromagnetic design. They have also been applied to synthesis of arrays [9]. Techniques as different as Simulated Annealing (SA), Genetic Algorithm (GA) and Particle Swarm Optimization (PSO) has been widely applied to different problems in antenna arrays. In the present work these techniques are used to find suitable amplitude excitations to generate required difference patterns.

\section{Methods}

\section{(a). Genetic Algorithm}

Genetic Algorithm is a robust, stochastic search method modeled on the concepts of natural selection and evolution. As an optimizer, GA is effective at solving complex, combinational and related problems, and is particularly effective when the goal is to find an approximate global maxima/minima in high dimension [9]. 
GA's operate on a group (or population) of trial solutions (or chromosomes) in parallel and they use simple stochastic operators (selection, crossover and mutation) to explore the solution domain [10]. In keeping with the natural world analogy, successive population of trial solutions are called generation. Subsequent generations are made up of children produced through selective reproduction of pairs of parents taken from the current generation.

The algorithm has following steps [11]-[12]

1. Create an initial population

2. Evaluate the fitness of each population member

3. Invoke natural selection

4. Select population members for mating (crossover)

5. Generate offspring

6. Mutate selected members of the population

7. Terminate run or go to step 2

Initial Population: It is set of chromosomes and each chromosome consists of genes (or variables) depend on number of variables. If each variable is constrained to be between zero and one then it is known as binary GA. If each variable takes a real value then it is known as real GA. In binary GA each variable is encoded with certain fixed number of bits.

Evaluate the fitness: Defining cost function is very important step in optimization. The chromosomes are passed to the cost function for evaluation. Each chromosome has an associated cost. If the cost function is defined to satisfy one goal then it is known as single-objective optimization. In some problems cost function must satisfy more than one goal. Such type of problem is known as multi-objective optimization.

Natural Selection: The healthy members of the population are allowed to survive to the next generation and discard the rest. Generally this can be done in two ways. In the first approach, the chromosomes are sorted on the basis of their associated cost. Only fixed number of chromosomes are retained and the rest are discarded. A second approach, called thresholding, keeps all chromosomes that have cost below a threshold cost value.

Mating: The most fit members of the population are assigned the highest probability of being selected for mating. Two chromosomes are selected from mating pool of chromosomes to produce two new offspring's. There are varieties of ways to select the mating chromosomes. Example; paring from top to bottom, Random pairing, Roulette- Wheel selection, tournament selection etc.

Generating offspring: Off spring can be generated from selected parents using different crossover methods, where crossover is an operator that forms a new chromosome from two parent chromosome by combining part of the information from each. Different crossover methods include single point crossover, multi point crossover, linear, quadratic, heuristic crossover etc.

Mutation: It is a process in which the values of genes are randomly altered in a parent chromosome. The mutation rate is the portion of bits or values within a population that will be changed. Example; A binary mutation changes a one to zero or a zero to one.

Termination: The generation process is repeated until a termination condition has been reached. The termination condition may be cost lower than minimum cost or set number of iterations or set time is reached.

\section{(b). Particle Swarm Optimization}

The Particle swarm optimization is a robust stochastic evolutionary computation based on the movement and intelligence of swarms. It was originated by imitating the behavior of a swarm of bees, a flock of birds or a school of fish during their food searching activities. It was developed by Eberhart and Kennedy in 1995. PSO has been shown to be effective in optimizing difficult multidimensional discontinuous problems in a variety of fields. With its advantages like simple structure, ease of implementation, speed and robustness it gained immediate popularity. Recently this technique has been successfully applied to antenna design [13].

In the PSO algorithm, particles are flown through a multidimensional search space, where the position of each particle is adjusted according to its own experience (pbest) and that of its neighbors (gbest). Updating the particles velocity is the core element of the entire optimization. The velocity of the particle is changed according to the relative location of the pbest and gbest [11].

$\mathrm{V}_{\mathrm{mn}}^{\text {new }}=\mathrm{V}_{\mathrm{mn}}^{\text {old }}+\mathrm{l}_{1} \times \mathrm{r}_{1} \times\left(\mathrm{P}_{\mathrm{mn}}^{\text {localbest }}-\mathrm{P}_{\mathrm{mn}}^{\text {old }}\right)+\mathrm{l}_{2} \times \mathrm{r}_{2} \times\left(\mathrm{P}_{\mathrm{mn}}^{\text {globalbest }}-\mathrm{P}_{\mathrm{mn}}^{\text {old }}\right)$

Where, $V_{\mathrm{mn}}=$ Particle velocity

$\mathrm{r}_{1}, \mathrm{r}_{2}=$ independent uniform random numbers

$1_{1}, l_{2}=$ learning factors

$\mathrm{P}_{\mathrm{mn}}$ localbest $=$ best local solution

$\mathrm{P}_{\mathrm{mn}}{ }^{\text {globalbest }}=$ best global solution

Once the velocity is updated it is easy to find next position of the particle.

$\mathrm{P}_{\mathrm{mn}}^{\text {new }}=\mathrm{P}_{\mathrm{mn}}{ }^{\text {old }}+\mathrm{V}_{\mathrm{mn}}{ }^{\text {new }}$ 
Where

$\mathrm{P}_{\mathrm{mn}}=$ particle position

If best local solution cost < best global solution cost, then replace current solution with best local solution. This process is carried out for each particle in the swarm.

\section{(c). Simulated Annealing}

Simulated Annealing belongs to the field of stochastic and Metaheuristics which is inspired by the process of annealing in metallurgy. SA simulates the behavior of the molecules of a pure substance during the slow cooling that results in the formation of a perfect crystal (minimum energy state). This process increases the strength and durability of the material [14].

SA was developed in 1983 to deal with highly nonlinear problems. It has been proved that by carefully controlling the rate cooling of temperature, SA can find the global optimum [15]. The algorithm employs a random search which not only accepts changes that decrease the objective function $\mathbf{f}$, but also some changes that increase it. So SA's major advantage over the other methods is an ability to avoid becoming trapped in local minima. The changes in the objective function are accepted with a probability of

$\mathrm{P}=\exp (-\delta \mathrm{f} / \mathrm{T})$

Where

$\delta \mathrm{f}$ is the increase in $\mathrm{f}$

$\mathrm{T}$ is control Parameter (Initial Temperature)

\section{Formulations}

The difference pattern from a continuous line source is also obtained from

$$
E_{d}(u)=\left[\int_{-1}^{o} A(x) e^{j \frac{j \pi L}{\lambda}[u x+\alpha]} d x+\int_{0}^{1} A(x) e^{j \frac{2 \pi L}{\lambda}[u x+\alpha]} d x\right]
$$

Here A $(\mathrm{x})$ is excitation function

$\frac{2 L}{\lambda}=$ Normalized array length

$\mathrm{u}=\sin \theta$

$\mathrm{x}=$ position on line source

$\alpha(\mathrm{x})=$ excitation phase

To generate a null in the boresight directions $180^{\circ}$ phase shift is introduced to one half of the array i.e.

$$
\begin{gathered}
\alpha=0 \text { for } \quad-1 \leq x \leq 0 \\
\alpha=\pi \text { for } \quad 0 \leq x \leq 1
\end{gathered}
$$

Substituting equation (2) in equation (1), then radiation pattern is now given by

$$
E(u)=\int_{-1}^{o} A(x) e^{j \frac{2 \pi L}{\lambda} u x} d x+\int_{0}^{1} A(x) e^{j \frac{2 \pi L}{\lambda}(u x+\pi)} d x
$$

Let $\mathrm{a}=\frac{2 \pi \mathrm{L}}{\lambda}$ then

$$
E(u)=\int_{-1}^{o} A(x) e^{j a u x} d x+\int_{0}^{1} A(x) e^{j a(u x+\pi)} d x
$$

$$
\mathrm{E}(\theta)=\mathrm{A}(\mathrm{x})\left\{\int_{-1}^{\mathrm{o}} \cos (\mathrm{aux})+\mathrm{j} \sin (\mathrm{aux}) \mathrm{dx}\right\}+\mathrm{A}(\mathrm{x})\left\{\int_{0}^{1} \cos (\operatorname{aux}+\pi)+\mathrm{j} \sin (\operatorname{aux}+\pi) \mathrm{dx}\right\}
$$

As the line source is only theoretical concept a discrete array is used in the present work. Here the radiation integral is replaced by a finite summation containing number of terms equal to the number of elements in the array.

The element locations are found out using Ishimaru spacing given by [16]

$$
\mathrm{x}=\frac{2 \mathrm{n}-1-\mathrm{N}}{\mathrm{N}}
$$

The radiation pattern is now given by (7)

$$
\begin{gathered}
\mathrm{E}(\mathrm{u})=\sum_{\mathrm{n}=1}^{\mathrm{N}} \mathrm{A}(\mathrm{x}) \mathrm{e}^{\mathrm{j} \frac{2-\mathrm{L}}{\mathrm{2}}[\mathrm{ux}+\mathrm{a}(\mathrm{x})]_{\mathrm{dx}}} \mathrm{dx} \\
\alpha=0 \text { for } \quad-1 \leq \mathrm{x} \leq 0 \\
\alpha=\pi \text { for } \quad 0 \leq \mathrm{x} \leq 1
\end{gathered}
$$

Where,

$\mathrm{A}(\mathrm{x})=1$ indicates that the source is uniformly excited. In a uniform array, deep null is at the boresight but the first side lobe level of the difference pattern is found to be $-11 \mathrm{~dB}$. These are considered to be inadequate for fine angular tracking radars. Sidelobe levels can be further reduced by carefully designing amplitude excitation 
or phase excitation or spacing between the elements of the array. In the present work, optimization of amplitude excitation coefficients of the array is considered using Genetic Algorithm (GA), Particle Swarm Optimization (PSO) and Simulated Annealing (SA).

\section{Results and Conclusions}

In this paper the authors used GA, PSO and SA to obtain difference pattern with deep null at the boresight and lowest possible sidelobe levels. The amplitude excitation coefficients obtained by applying above optimization techniques are shown in table 1 for a typical $\mathrm{N}=30$ element array. The same can extended for large and small arrays. The patterns are numerically computed and are shown in $\sin \theta$ (or $u$ ) domain in figures (1-3).

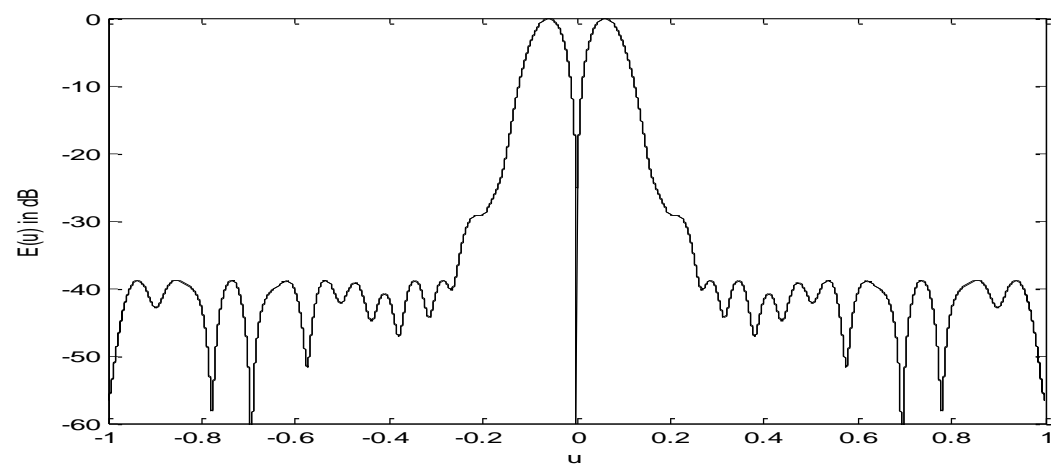

Fig. 1. Difference Pattern for $\mathrm{N}=30$ elements optimized using Genetic Algorithm (GA).

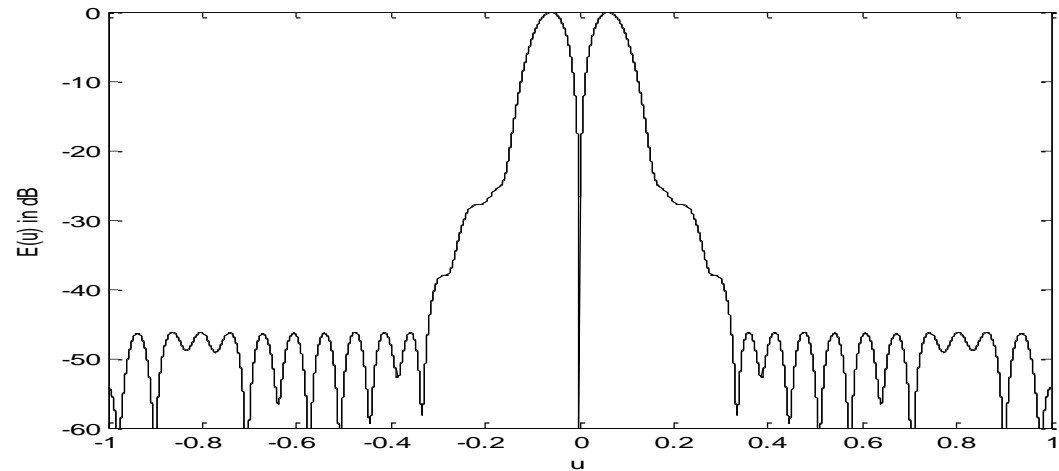

Fig. 2. Difference Pattern for $\mathrm{N}=30$ elements optimized using Particle Swarm Optimization (PSO)

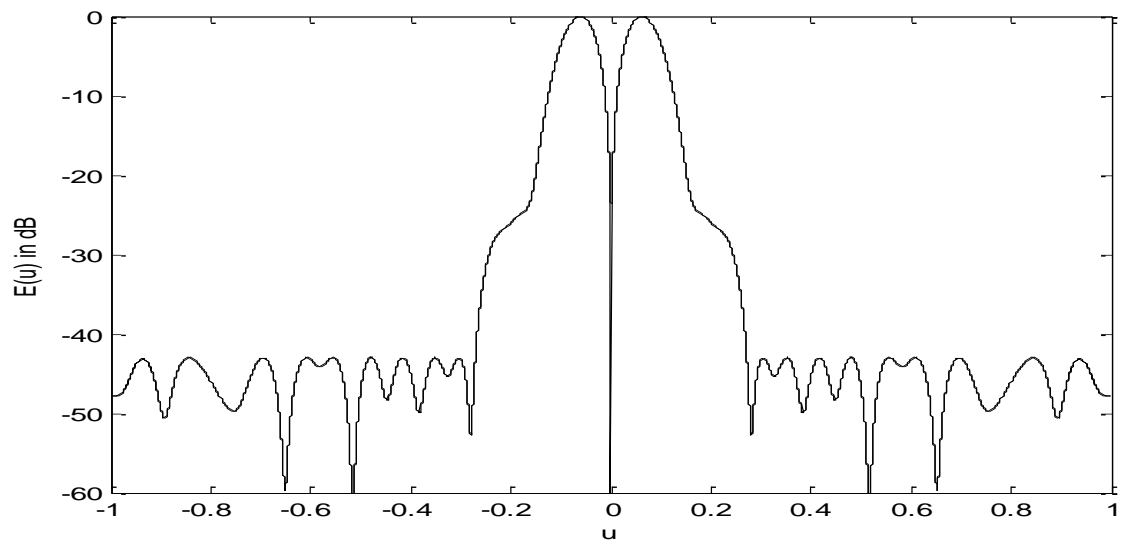

Fig. 3. Difference Pattern for N=30 elements optimized using Simulated Annealing (SA). 


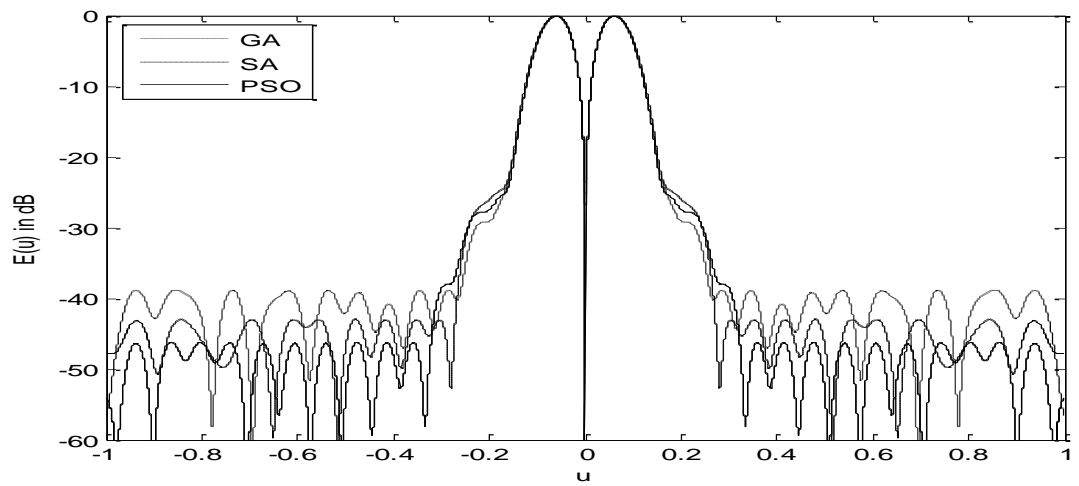

Fig. 4. Overlap of Difference Pattern optimized by GA, SA \& PSO methods for $\mathrm{N}=30$ elements

\begin{tabular}{|c|c|c|c|c|}
\hline $\begin{array}{c}\text { No. of elements } \\
N\end{array}$ & $\begin{array}{c}\text { Spacing } \\
\text { Position } \\
x_{n}\end{array}$ & $\begin{array}{c}\text { Amplitude } \\
\text { Distribution } A\left(x_{n}\right) \\
\text { GA }\end{array}$ & $\begin{array}{c}\text { Amplitude } \\
\text { Distribution } A\left(x_{n}\right) \\
\text { PSO }\end{array}$ & $\begin{array}{c}\text { Amplitude } \\
\text { Distribution } A\left(x_{n}\right) \\
S A\end{array}$ \\
\hline 1 & -0.9667 & 0.0855 & 0.0806 & 0.0639 \\
\hline 2 & -0.9000 & 0.1404 & 0.1329 & 0.1322 \\
\hline 3 & -0.8333 & 0.2492 & 0.2397 & 0.2242 \\
\hline 4 & -0.7667 & 0.3755 & 0.3694 & 0.3374 \\
\hline 5 & -0.7000 & 0.5016 & 0.5037 & 0.4779 \\
\hline 6 & -0.6333 & 0.6787 & 0.6629 & 0.6223 \\
\hline 7 & -0.5667 & 0.7883 & 0.7918 & 0.7563 \\
\hline 8 & -0.5000 & 0.9183 & 0.9124 & 0.8893 \\
\hline 9 & -0.4333 & 1.0000 & 0.9912 & 0.9338 \\
\hline 10 & -0.3667 & 0.9920 & 1.0000 & 1.0000 \\
\hline 11 & -0.3000 & 0.9487 & 0.9528 & 0.9333 \\
\hline 12 & -0.2333 & 0.8365 & 0.8205 & 0.8090 \\
\hline 13 & -0.1667 & 0.6292 & 0.6246 & 0.6333 \\
\hline 14 & -0.1000 & 0.3766 & 0.3744 & 0.3448 \\
\hline 15 & -0.0333 & 0.1027 & 0.0868 & 0.0421 \\
\hline 16 & 0.0333 & 0.1920 & 0.2014 & 0.2519 \\
\hline 17 & 0.1000 & 0.4952 & 0.4771 & 0.4932 \\
\hline 18 & 0.1667 & 0.6666 & 0.6878 & 0.6919 \\
\hline 19 & 0.2333 & 0.8719 & 0.8421 & 0.8379 \\
\hline 20 & 0.3000 & 0.9220 & 0.9163 & 0.9153 \\
\hline 21 & 0.3667 & 0.9856 & 0.9438 & 0.9307 \\
\hline 22 & 0.4333 & 0.9436 & 0.9169 & 0.9266 \\
\hline 23 & 0.5000 & 0.8871 & 0.8840 & 0.8719 \\
\hline 24 & 0.5667 & 0.8160 & 0.8176 & 0.8101 \\
\hline 25 & 0.6333 & 0.7049 & 0.7333 & 0.7153 \\
\hline 26 & 0.7000 & 0.6314 & 0.6322 & 0.6140 \\
\hline 27 & 0.7667 & 0.4782 & 0.4888 & 0.4424 \\
\hline 28 & 0.8333 & 0.3686 & 0.3454 & 0.3246 \\
\hline 29 & 0.9000 & 0.2824 & 0.2102 & 0.1958 \\
\hline 30 & 0.9667 & 0.1568 & 0.0904 & 0.0785 \\
\hline
\end{tabular}

Table 1 : Distributions for $\mathrm{N}=30$ Elements

\section{Acknowledgements}

I, T.A.N.S.N.Varma, thank Maharaj Vijayaram Gajapathi Raj College of Engineering, Vizianagaram for the support for my Ph.D work.

\section{References}

[1]. G.S.N.RAJU. "Antennas and Wave Propagation". Pearson Education, 2005.

[2]. M.T.Ma, "Theory and application of Antenna Arrays", John Wiley \& Sons Inc, 1974.

[3]. D Mandal, S K Ghosal, S Das, S Bhattacharjee and A K Bhattacharjee, “ Improvement of radiation pattern for linear antenna arrays using genetic algorithm," 2010 international conference on recent trends in Information, Telecommunications \& Computing.

[4]. E.T.Bayliss, "Design of monopulse antenna difference patterns with low sidelobes," Bell Syst. Tech. J., vol. 47, pp. 623-650, MayJune 1968 .

[5]. R.S.Elliott, "Design of line source antennas for difference patterns with sidelobes of individually arbitrary heights," IEEE Transactions on Antennas and Propagation, vol. AP-24, no. 3, pp.310-316, 1976. 
[6]. Yanchang, G.; Meng, M.; Xianrong, S.; Nenghang, F., "Design of aperture distributions for difference pattern from Taylor distributions," Antennas and Propagation Society International Symposium, 1993. AP-S. Digest, vol., no., pp.1586,1589 vol.3, June 28 1993- July 21993.

[7]. YanChang Guo; Meng Miao, "Design of antenna array aperture distributions for difference pattern using Dolph-Chebyshev distribution," Microwave Conference Proceedings, 1993. APMC '93., 1993 Asia-Pacific , vol.1, no., pp.1-58,1-61 vol.1, 1993.

[8]. Salvatore Caorsi, "Optimization of difference patterns for monopulse antennas by a hybrid real/integer coded differential evolution method," IEEE Transactions on Antennas and Propagation, vol. 53, no. 1, pp.372 -376, January 2005.

[9]. Johnson, J. M. and Y. Rahmat-Samii, "Genetic Algorithm optimization and its applications to Antenna Design", IEEE Transactions on Antennas and Propagation, vol. 39, no. 4, pp.326 -329, 1994

[10]. Johnson, J. M. and Y. Rahmat-Samii, "Genetic Algorithms Optimization of Wireless Communication Networks," IEEE Antennas and Propagation Society International Symposium, June 18-23, 1995, pp. 1964- 1967.

[11]. R.L.Haupt,"An Introduction to Genetic Algorithms for Electromagnetics", IEEE Antennas and Propagation Magazine, vol. 37, no. 2, pp.7 -15 1995 .

[12]. R. L. Haupt, Douglas H. Werner . "Genetic Algorithms in Electromagnetics”. John Wiley \& Sons, 2007.

[13]. Nanbo Jin, Y. Rahmat-Samii, "Advances in Particle Swarm Optimization for Antenna Designs: Real-Number, Binary, SingleObjective and Multiobjective Implementations," IEEE Transactions on Antennas and Propagation, vol. 55, no. 3, pp.556 -567, 2007.

[14]. Peter J. Bevelacqua, Constantine A. Balanis, "Optimizing Antenna Array Geometry for Interference Suppression," IEEE Transactions on Antennas and Propagation, vol. 55, no. 3, pp.637 -641, 2007.

[15]. Randy L. Haupt, Sue Ellen Haupt. "Practical Genetic Algorithms". 2e, John Wiley \& Sons, 2007.

[16]. Ishimaru, "Theory of unequally spaced arrays," IRE transactions on Antennas and Propagation, Vol. AP-10, pp. 691-702, November 1962.

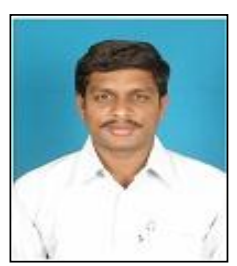

\section{Author's Information}

Mr. T.A.N.S.N.Varma received his B.Tech in Electronics \& Communication Engineering from JNTU, Hyderabad in 2005 and his M.Tech in Radar \& Microwave Communication from Andhra University in 2008. Presently working for his Ph.D in Andhra University under the guidance of Prof. G.S.N.Raju. His areas of interests are Antenna arrays and EMI/EMC. Mr. Varma is a member of IEEE, IETE, ISTE and SEMCE.

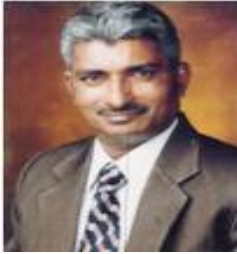

Dr. G.S.N. Raju received his B.E., M.E. with distinction and first rank from Andhra University and Ph.D. from IIT, Kharagpur. He is presently Vice-Chancellor, Andhra University Visakhapatnam, India. He is in teaching and research for the last 30 years in Andhra University. He guided 29 Ph.D.s in the fields of Antennas, Electro Magnetics, EMI/EMC and Microwave, Radar Communications, Electronic circuits. Published about 300 technical papers in National/International Journals/Conference Journals and transactions. He is the recipient of The State Best Teacher Award ${ }^{\text {ee }}$ from the Government of Andhra Pradesh in 1999, "The Best Researcher Award"e in 1994, "Prof. Aiya Memorial National IETE Award"e for his best Research guidance in 2008 and Dr. Sarvepalli Radhakrishnan Award for the Best Academician of the year 2007, He was a visiting Professor in the University of Paderborn and also in the University Karlsruhe, Germany in 1994. At present he holds the positions of Vice-Chancellor, Andhra University, Visakhapatnam. He was Chief Editor of National Journal of Electromagnetic Compatibility. Prof. Raju has published five textbooks Antennas and Wave Propagation, Electromagnetic Field Theory and Transmission Lines, Electronics Devices and Circuits, Microwave Engineering, Radar Engineering and Navigational Aids. Prof. Raju has been the best faculty performer in Andhra University with the performance index of 99.37. 